\title{
La Comunicación Social en el Ecuador, elementos para repensar su historia
}

\section{Social Communication in Ecuador, elements to rethink its history}

DOI: https://doi.org/10.29166/tyc.v1i20.2124

\section{Patricio Pilca}

Cursa estudios de doctorado en Ciencias Sociales en la Universidad de Buenos Aires (Argentina); Magíster en Sociología por la Facultad Latinoamérica de Ciencias Sociales (FLACSO-Ecuador); Sociólogo por la Universidad Central del Ecuador. Tiene experiencia docente en Educación Superior, en asignaturas como: Historia, Filosofía, Realidad Ecuatoriana, Teoría Social y Política, y Pensamiento Económico, entre otras. Ha trabajado en el desarrollo de proyectos educativos relacionados con la universidad pública, culturales e históricos.

Correo: eppilca@uce.edu.ec

\section{Resumen}

El presente trabajo indaga en la historia de la Facultad de Comunicación Social (FACSO) de la Universidad Central del Ecuador (UCE) desde la década de 1930 hasta 1983. En este período se observan los distintos momentos por los cuales han transitado los estudios de comunicación, primero como cátedra en 1938; luego como Escuela de Periodismo en 1945; como Ciencias de la Información en 1963; y finalmente, en 1983, como Facultad de Comunicación Social. Para este trabajo se revisaron los archivos de la UCE, sobre todo, las actas del Honorable Consejo Universitario (HCU) de la década de 1930. Para las décadas posteriores se recabó información de documentos históricos de la propia FACSO, especialmente, los planes de carrera. Finalmente, este artículo sostiene que los estudios de comunicación no se iniciaron en 1945, como muchos aseveran, sino en 1938, como parte de una carrera universitaria.

Palabras clave: historia de la comunicación, escuela de periodismo, ciencias de la información, FACSO Ecuador.

\section{Abstract}

This work researches the history of the Faculty of Social Communication (FACSO) of Central University of Ecuador (UCE) from the 1930s to 1983. In this period, the different moments communication studies have traveled are observed, first as a chair in 1938; then as a School of Journalism in 1945; as Information Sciences in 1963; and finally, in 1983, as a Faculty of Social Communication. For this work, the files of the UCE were reviewed, especially the minutes of the Honorable University Council (HCU) of the 1930s. For subsequent decades, information was collected from historical documents of the FACSO itself, especially career plans. Finally, this article maintains that communication studies were not started in 1945, as many claim, but in 1938, as part of a university career.

Keywords: history of communication, journalism school, information science, FACSO Ecuador. 
El archivo se presenta así como un lugar físico que aloja el destino de esta especie de huella que, con todo cuidado, nosotros distinguimos de la huella cerebral y de la huella afectiva, es decir, la huella documental.

Paul Ricœur La memoria, la historia y el olvido

\section{Introducción}

A partir del archivo, que contiene indicios y huellas, se puede reflexionar sobre la historia. En este sentido, la recuperación que se pueda hacer de esta última, potencia la vida de las instituciones. El siguiente artículo explora en fuentes históricas acerca del origen de la Comunicación Social -como disciplina académica- en la Universidad Central del Ecuador (UCE).

Este escrito es producto de tres esfuerzos conjuntos: en primer lugar, el proyecto de investigación Reconstrucción de la memoria histórica de la Facultad de Comunicación Social (FACSO) de la Universidad Central del Ecuador, ejecutado en 2017, como parte de las prácticas preprofesionales $^{1}$ que los estudiantes realizan para graduarse de comunicadores sociales; en segundo lugar, las discusiones en el marco de las "I Jornadas de Co- municación”, realizadas en la FACSO en 2019; y, finalmente, el aporte de la tesista Ariana Román, que se basa en la revisión de anteriores mallas curriculares de la misma facultad.

Si bien existen algunas investigaciones en torno a la historia de la facultad, muchas carecen de fuentes bibliográficas, lo que ha llevado a confusiones interpretativas. Por ello, este artículo muestra la genealogía de la institución, a partir de un trabajo de archivo. Esto implicó la revisión metodológica de materiales históri$\cos ^{2}$, principalmente actas del Honorable Consejo Universitario (HCU) de la Universidad Central del Ecuador (UCE), archivos de la facultad y entregas del Registro Oficial.

En resumen, los primeros antecedentes de la Facultad de Comunicación Social datan de 1937, cuando se discutió la creación de la cátedra de Periodismo, en la entonces Facultad de Pedagogía y Letras, que dio paso a la posterior creación de la Escuela de Periodismo, años más tarde. En 1944, en el rectorado de Julio Paredes, el HCU discutió acerca de la posibilidad de la anexión de instituciones para la posible reaparición de la Facultad de Filosofía, que podía incluir tres carreras: la de Filosofía y Pedagogía, la de Periodismo y Letras y la Escuela o Sección de Estudios Históricos. En 1945se publicó el decreto número 315 en el Registro Oficial, indicando así la creación de las Escuelas de Periodismo, en las

1 El proyecto "Reconstrucción de la memoria Histórica de la Facultad de Comunicación Social (FACSO) de la Universidad Central del Ecuador", realizado en el año 2017, surgió como parte de un cuestionamiento acerca del origen y los cambios que ha tenido la carrera, desde su creación. Este proyecto busca investigar, identificar y describir la historia de la facultad; en última instancia busca construir una genealogía de la institución.

2 El trabajo de archivo estuvo sujeto a dos momentos: en el primero se revisó el periodo comprendido entre 1938 -1948 y en el segundo entre 1948-1968. En ambos periodos se encontró dos tipos de actas, fechadas con el mismo día, año y número de reunión, sin embargo, su contenido no es igual, más bien parece que ambos documentos completan la información de la toda la sesión. Es una especie de complemento entre ambas actas, donde se detallan ciertos elementos que en primera instancia no se lograron recoger. 
Universidades de Quito y Guayaquil. El decreto fue expedido por el Congreso Nacional y firmado por el presidente José María Velasco Ibarra. En las sesiones del HCU de 1946 se discutió el Reglamento y el Plan de Estudios de la Escuela de Periodismo. Años más tarde, en 1963, se creó la Escuela de Ciencias de la Información. Finalmente, en 1983 se creó la Facultad de Comunicación Social.

Como todo proyecto de investigación, este todavía debe alimentarse con los aportes de profesores, estudiantes y cuerpo administrativo. Este artículo es una primera aproximación que coloca a la facultad, donde cotidianamente se desenvuelven las actividades universitarias y la comunicación como disciplina, como un objeto de estudio. Es hora de volver los ojos hacia las instituciones donde un sin número de personas trabaja, para conocer nuestro pasado, más aún, es necesario regresar al trabajo de archivo como posibilidad investigativa que involucra a la comunicación directamente.

\section{La comunicación en el "corto siglo XX"}

La Facultad de Comunicación Social (FACSO-Quito) ha transcurrido por cuatro momentos institucionales en su constitución como tal: primero, en la década de 1930, como catedra; segundo, en 1945, como Escuela de Periodismo; tercero, en la década de 1960, con la creación de la Escuela de Ciencias de la Información; y, finalmente, en la década de 1980, con la creación de la Facultad de Comunicación Social. A lo largo de los estos periodos se manejaron dieciocho planes de estudios, cada uno con un discurso comunicacional que marcaba una línea académica diferente en cada etapa.
La comunicación en el siglo XX, tuvo un contexto político complejo en el Ecuador. De acuerdo con Wilson Hallo (1992, p. 97), con la emergencia del liberalismo, el país dio un giro determinante, sobre todo por los avances políticos, sociales, económicos y comunicacionales. "El progreso de la nación fue palpable y la efervescencia por (...) la cultura se nota por medio del desarrollo del periodismo, que de una manera inusitada sobresale en el Ecuador" (Hallo, 1992, p. 97). Las personas que ejercieron el periodismo a finales del siglo XIX e inicios del XX eran personas ilustres que informaban sobre temas políticos y de opinión de forma más bien empírica. En las décadas del treinta y cuarenta del siglo XX se trató de innovar e implementar una matriz profesionalizante.

La profesionalización de la comunicación surgió con la creación de las Escuelas de Periodismo en casi toda América Latina, en la década de 1930. A partir de la segunda posguerra, se fundaron las escuelas por la urgencia de los grandes medios de comunicación de modernizar el sistema de medios impresos, lo que obligó a pensar en la profesionalización de los periodistas en las universidades. En el caso ecuatoriano, con el afán de realizar esto y organizar a los periodistas, se creó la Unión Nacional de Periodistas (UNP) en 1944. Con el establecimiento de esta institución, el periodismo inició una nueva etapa con bases profesionales y técnicas.

En el Ecuador, las Escuelas de Periodismo aparecieron por decreto legislativo de la Asamblea Nacional Constituyente, el 8 de marzo de 1945. Bajo este argumento se piensa que la primera escuela se creó en Quito en aquel año, y la segunda, un año más tarde, en la Universidad Estatal de Guayaquil. Por tanto, en las pocas investigaciones que trabajan con 
la memoria institucional ${ }^{3}$ aparece 1945 como el año en que inicia el periodismo en el país. Así, por ejemplo, Albalira Pérez de Ricaurte (1975, p. 2) sostiene que los señores Jorge Reyes y Miguel Albornoz ${ }^{4}$ fueron los que "presentaron a la Asamblea Nacional Constituyente de 1945, un Proyecto de Decreto de Fundación de las Escuelas de Periodismo de Quito y Guayaquil, habiendo obtenido la aprobación de la Asamblea por unanimidad el día 8 de marzo de 1945”. En esta misma línea, Víctor Hugo Escobar y Edison Carlos Ramírez Tarapuez (1993, p. 1) manifiestan que la trayectoria de la FACSO inició en el año 1945. En el año 2005, Juan García González (2005, p. 125), ex decano, manifiesta que "La actual Facultad de Comunicación Social de la Universidad Central del Ecuador, tuvo su origen en un Curso de Periodismo, tipo extensión universitaria, organizado por la Facultad de Filosofía, Letras y Ciencias de la Educación durante los años 1942 y 1943”. También hay un texto de 2009, elaborado por Fernando Maldonado Donoso, ex profesor de la Facultad de Comunicación de la Universidad Central, donde sostiene que, en el año 1945, se creó la Escuela de Periodismo (Maldonado, 2009, p. 71).

En todos estos trabajos se sitúa 1945 como el año de creación de las escuelas de periodismo. Lo sintomático es que en las investigaciones de Albalira Pérez, así como en la de Juan García González, se habla de unos cursos de perio- dismo, ahí es donde está investigación pretende explotar un poco más. Lo que permite poner en cuestión esta fecha e indaga antecedentes de dichos cursos en la década del treinta del siglo pasado. Esto aporta nuevos elementos históricos a la discusión historiográfica de la FACSO.

\section{De la cátedra a la facultarización: 1938-1983}

En esta sección se reconstruirá la historia de la Facultad de Comunicación Social, en base a la investigación realizada y los documentos encontrados en el Archivo Histórico (se utilizará las actas ${ }^{5}$ del HCU del periodo comprendido entre 1938 y 1948) de la Universidad Central del Ecuador, así como archivos de la misma facultad y documentos del Registro Oficial, sobre todo en las décadas de 1930 y 1940. Para las décadas de 1960 y 1980 se revisaron los Planes Directores de la carrera.

\section{Décadas de 1930 y 1940}

Uno de los primeros documentos relacionados a la historia de la Facultad de Comunicación tiene fecha 14 de noviembre de 1938. En este documento se mencionan la creación de la cátedra de Periodismo en la Facultad de Pedagogía y Letras. Las autoridades que asistieron a esa sesión fueron: Gualberto Arcos, Rector; Rafael A. Cruz, vicerrector; Gustavo

3 Sobre todo, trabajos de tesis realizados por alumnos (as) para la obtención del título profesional, así como algunos artículos realizados por ciertos profesores, presentados en revistas de la misma facultad.

4 Según la investigación de Albalira Pérez, estas dos personas, en los años 1943-44, fueron los primeros directores de los "Primeros Cursos Libres de Periodismo" en Quito, acogiéndose a los ciclos de Extensión Cultural de la Universidad Central (1975:2)

5 En el periodo 1938-1948, sobresale la presentación de dos actas por cada sesión de HCU. Cabe mencionar que este órgano colegiado, normalmente, sesionaba dos veces por mes. 
Buendía, decano de Jurisprudencia; Carlos Bustamante Pérez, decano de Medicina; Manuel García, representante del Ministerio de Educación Pública y los señores Manuel de Guzmán (suplente), Leopoldo Arcos y Enrique Unda, representantes estudiantiles por Jurisprudencia, Medicina y Pedagogía, respectivamente (Acta de Sesión Honorable Consejo Universitario de 14 de noviembre de 1938 A, 1938).

En esta acta aparece, por primera vez, la preocupación por pensar el periodismo en la universidad pública. El acta registra que Gualberto Arcos, entonces rector de la Universidad Central del Ecuador, sugiere al HCU "la conveniencia de que cuanto antes, se convierta en realidad el establecimiento de la Escuela de Periodismo $^{6}$, en la Facultad de Pedagogía y Letras" y además indica que "debería pedirse a la respectiva junta de Facultad la terna correspondiente para el nombramiento de profesor de la indicada catedra" (Acta de Sesión Honorable Consejo Universitario de 14 de noviembre de 1938 $A, 1938$, f. 96). El Consejo acepta la petición del señor rector y dispone que, a través de secretaría, se pida a la facultad la mencionada terna. El documento es un tanto confuso ya que no es claro si se habla de inaugurar una cátedra o más bien la Escuela de Periodismo.
Sin embargo, en la segunda acta de la misma sesión sí se menciona la creación de la Escuela de Periodismo. En esta segunda acta aparecen más detalles, que permiten ampliar la mirada interpretativa. El acta dice lo siguiente:

El señor Rector antes de dar a conocer el orden del día, pone en conocimiento del consejo el siguiente asunto: "[...] en el año anterior [1937] en una de las sesiones del consejo, se trató acerca de la creación en la Facultad de Pedagogía, de la Escuela de Periodismo y, para realizarlo, lo único que nos hace falta es el profesor de esta materia; para esto, sería del caso dirigirse a la junta de la facultad, solicitándole la respectiva terna para el nombramiento de profesor de la indicada catedra" (Acta de Sesión Honorable Consejo Universitario del 14 de noviembre de 1938 B, 1938, f. 101).

Hasta aquí lo novedoso es la mención del año 1937. Pero además, el señor Enrique Unda7, representante estudiantil, menciona que "esta escuela [de Periodismo] va a pertenecer a la Facultad de Pedagogía" (Acta de Sesión Honorable Consejo Universitario del 14 de noviembre de 1938 B, 1938, f. 101); más adelante indica que "los estatutos dicen que el plan de estudios debe ser aprobado por el Consejo Directivo de la facultad, es decir, que los mismos programas pasan primeramente a conocimiento del Consejo Direc-

6 El resaltado es mío.

7 En la misma acta se menciona los requisitos para ser un estudiante universitario. Como esta escuela va a pertenecer a la Facultad de Pedagogía, los alumnos tienen que sujetarse a lo dispuesto en el art. 65 de la ley de Educación superior. El Sr. secretario da lectura al mencionado artículo en el que dice que para ingresar a la universidad se requiere:

- $\quad$ Primero: tener por lo menos 18 años de edad

Segundo: presentar los certificados de estudio, título de bachillerado y los demás que exigieren los Estatutos y Reglamentos. Tercero: Haber cumplido las obligaciones militares.

Cuarto: satisfacer las pruebas de capacidades exigidas en los estatutos y reglamentos.

- Quinto: pagar los derechos fijados en los reglamentos (Acta de Sesión Honorable Consejo Universitario de 14 de noviembre de 1938 A, 1938). 
tivo y luego son aprobados por el Consejo Universitario" (Sesión de 14 de noviembre de 1938: folio 102). Estas afirmaciones permiten sospechar que la creación de la escuela se planificaba para un futuro no muy lejano. A lo anteriormente mencionado se puede sumar la siguiente aseveración del rector Gualberto Arcos: "Las clases serán dictadas tanto por los profesores de la Facultad de Pedagogía, como por los de jurisprudencia; lo único que necesitamos es hacer el nombramiento del profesor de esta materia y abrir las matrículas" (Acta de Sesión Honorable Consejo Universitario del 14 de noviembre de 1938 B, 1938, f. 102).

A partir de estas declaraciones se puede observar que la creación, ya sea de la materia o de la escuela, está a puertas de cumplirse y que faltaban mínimos detalles para hacer posible su creación. Esta hipótesis se confirma con la intervención de Manuel García, representante por el Ministerio de Educación Pública, quien pidió que se formulara un programa para este curso, para el conocimiento del HCU (Acta de Sesión Honorable Consejo Universitario del 14 de noviembre de 1938 B, 1938). Las fuentes no son concluyentes en cuanto al formato de los estudios que la Universidad ofreció: se nombran Cursos de Periodismo, pero en su pensum también se incluyó asignaturas que pudieron haber sido cubiertas por docentes de las carreras de Pedagogía y Derecho. En cualquier caso, los cursos a los cuales hace alusión Juan García (2005), no son de los años 1942 o 1943, sino que tienen sus antecedentes en el año 1937 y se materializaron un año después, en 1938. De acuerdo con las fuentes, la Escuela de Periodismo inició como un curso dentro de la Facultad de Pedagogía, con todo un plan de estudios, que creó los fundamentos para las posteriores instituciones que asumieron la enseñanza de esta disciplina.

La creación de la Escuela de Periodismo era un objetivo que no perdían de vista las autoridades. Se podría afirmar que los cursos y la creación de la escuela van de la mano. Esto es más claro en el acta de la sesión del 25 de noviembre de 1938. A esta acuden: Gualberto Arcos, rector; Rafael A. Cruz, vicerrector; Gustavo Buendía, decano de Jurisprudencia; Carlos Bustamante Pérez, decano de $\mathrm{Me}$ dicina; Jorge Escudero, decano de Pedagogía; Pablo Palacio, representante de la Asamblea Universitaria; Manuel García, representante por el Ministerio de Educación Pública; los señores: Manuel de Guzmán (suplente), Leopoldo Arcos, Gustavo Jaramillo y Enrique Unda, representantes estudiantiles por Jurisprudencia, Medicina, Ciencias y Pedagogía, respectivamente. Concurre también Alberto Batallas, tesorero del plantel. En esta sesión, el HCU, encabezado por el señor rector, Gualberto Arcos, declaró: "establecida en la Facultad de Pedagogía y Letras, la Escuela de Ciencias para Profesores de Segunda Enseñanza; como declara también igualmente establecida en la propia facultad la Escuela de Periodismo, debiendo proveerse únicamente el cargo de Profesor de Técnica Periodística, propuesta en terna de la Facultad" "Acta de Sesión Honorable Consejo Universitario del 25 de noviembre de 1938 A, 1938, f. 115). A partir de esta información, se

8 El resaltado es mío. 
puede manifestar que la profesionalización del periodismo se gestó desde el año 1937, varios años antes de la creación formal de la carrera, mencionada en las investigaciones realizadas anteriormente.

Entre 1938 y 1943, en las actas de HCU no se nombra nada respecto de la Facultad de Comunicación o la Escuela de Periodismo, se asume que los cursos de periodismo se mantuvieron durante todo este tiempo. Es apenas en 1944 cuando aparece información en torno a esta escuela. En ese momento la discusión gira alrededor de la anexión de tres instituciones a la universidad, una de ellas es la Escuela de Periodismo. Hay dos reuniones de HCU en 1944 en que se retoman las discusiones sobre la Escuela de Periodismo; la primera en julio y la segunda en septiembre.

A la primera reunión, fechada el 25 de julio de 1944, asistieron: Julio E. Paredes, rector; los doctores Julio Endara y Augusto Estupiñán; los ingenieros Jorge Casares y Alfredo Reyes; y los representantes estudiantiles: Manuel Oña Silva y Germán Andrade. En actas se apunta la anexión de tres instituciones a la Universidad, sin indicar cuales son estas: "Por indicación del señor presidente, léase el decreto ejecutivo N. 409, de 14 de julio, [donde se publica lo siguiente] (...) Según el registro oficial $\mathrm{N}^{\circ} 41$, el 19 de los propios mes y año, por el que se anexan a la Universidad varias instituciones" (Acta de Sesión Honorable Consejo Universitario del 25 de julio de 1944 A, 1944a, f. 402). Este decreto fue sorpresivo para el señor presidente, pues no habían sido consultados ni el rector de la Universidad, ni los directores de los institutos anexados.

Esta información fue mejor detallada en la sesión de septiembre, cuyas actas mencionan que las instituciones que se anexarían eran el Instituto de Pedagogía, el Conservatorio de Música y la Escuela de Bellas Artes (Acta de Sesión Honorable Consejo Universitario del 25 de septiembre de $1944 A, 1944 b)$. A la reunión del 25 de septiembre de 1944 asistieron: Julio Endara, vicerrector, encargado del rectorado ${ }^{9}$ y quien preside esa reunión; y concurren los doctores Julio E. Paredes, rector, Augusto Estupiñán, Alberto Arroyo, Manuel Villacís; los ingenieros Jorge Casares y Alfredo Reyes; y los representantes estudiantiles: Manuel Oña Silva y Germán Andrade. Como invitados especiales acuden el vicerrector del Instituto de Pedagogía y los directores del Conservatorio de Música y de la Escuela de Bellas Artes.

En aquella sesión, el rector, Julio E. Paredes, expuso dos puntos importantes que, al parecer, le obligaron a estar en esa reunión, pues en ese momento su trabajo estaba centrado en el presupuesto de la universidad en la Asamblea Nacional, y no iba a acudir, por eso, quien preside esa reunión es el vicerrector, Dr. Julio Endara. El primer punto que expone el rector estaba relacionado con el presupuesto para la universidad, ya que él era miembro de la Comisión del Presupuesto de la Asamblea. El segundo punto que trató fue el relacionado con la anexión de los institutos a la universidad. Al respecto menciona que este decreto fue sorpresivo y

9 El Sr. Rector, Julio E. Paredes, encarga su cargo al Vicerrector, Sr. Dr. Julio Endara, ya que el rector, representante de la asamblea Universitaria, sería parte de la Asamblea Constituyente (Sesión del 25 de julio de 1944, folio 408). 
añade "Que no fueron consultados ni el rector, ni los directores de aquellos institutos. Que le pareció conveniente la resolución del Consejo Universitario" (Acta de Sesión Honorable Consejo Universitario del 25 de septiembre de 1944 A, 1944b, f. 416). En dicha resolución se menciona que los institutos deben funcionar con autonomía "hasta que sea estudiada detenidamente su nueva organización dentro de la entidad universitaria" (Acta de Sesión Honorable Consejo Universitario del 25 de septiembre de 1944 A, 1944b, f. 416). Y además menciona las ventajas y desventajas de dicha anexión. En cuanto a las ventajas sostiene que los estudiantes que asisten a estas instituciones ya no aprenderán a "merced de la política", mantendrán su autonomía y serán parte de la universidad (Acta de Sesión Honorable Consejo Universitario del 25 de septiembre de 1944 A, 1944b, f. 416). Entre las desventajas, manifiesta sólo una: la dependencia económica que mantendrán los institutos respecto de la universidad puede ser perjudicial, sobre todo por la estrechez del presupuesto (Acta de Sesión Honorable Consejo Universitario del 25 de septiembre de 1944 A, 1944b, f. 416)..

De acuerdo con el rector, con la Escuela de Bellas Artes se podría formar una nueva facultad como las que hay en las grandes universidades y que tendría un valor cultural trascendental para el país. A esta facultad pertenecería el Conservatorio. En cuanto al Instituto de Pedagogía, dice: "ya tiene su pasado en la Universidad cuando constituyó la Facultad de Filosofía y Letras cuya abolición fue hecha por la dictadura ${ }^{10}$. Cree que, de volverse a constituir la Facultad, ésta podría incluir tres escuelas: la de Filosofía y Pedagogía, la de Periodismo y Letras ${ }^{11}$ y la Escuela o sección de Estudios Históricos" (Acta de Sesión Honorable Consejo Universitario del 25 de septiembre de 1944 A, 1944b, f. 416-417).

Además, el rector planteó tres sugerencias que elevó a moción: 1) que los institutos elaborasen sus proformas concretas; 2) que se les faculta abrir los cursos correspondientes en el tiempo y la forma en que acostumbren; y 3) que se constituyese una comisión que estudie la organización de la Facultad de Filosofía y Letras. Además, apareció una cuarta sugerencia, planteada por el ingeniero Casares, que consistió en que "la Universidad formule una exposición extensa de motivos en torno a los problemas de la educación nacional, especialmente la universitaria, haciendo resaltar el abandono económico en que se ha venido manteniendo" (Acta de Sesión Honorable Consejo Universitario del 25 de septiembre de 1944 A, 1944b, f. 418). En la sesión de HCU se aceptaron las mociones planteadas y se manifestó que para la exposición de motivos se juntasen las siguientes personas: Alberto Arroyo, Jorge Casares, el señor Oña y el señor secretario. Mientras que para la organización de la Facultad de Filosofía y Letras se designó al vicerrector de la Universidad Central, Julio Endara; vicerrector del Instituto de Pedagogía; y German Andrade, representante de los estudiantes. La eficiencia con la que actúa el HCU indica una clara vo-

\footnotetext{
10 En la década del cuarenta no se registran dictaduras militares, más bien se dio el segundo Velasquismo (1944-1947), el cuál desconoció la constitución de La Gloriosa de 1944.

11 El resaltado es mío.
} 
luntad de que la Escuela de Periodismo sea parte de la Facultad de Filosofía, así como una intención de brindar una oferta educativa más variada, que se concentra en el campo de las humanidades.

En las décadas de 1930 y 1940 se dan algunas discusiones en torno al periodismo y la comunicación, donde sobresalen los cursos de periodismo y las múltiples discusiones en el seno del HCU, todas estas pensando el periodismo como profesión. Por iniciativa de Jorge Reyes y Miguel Albornoz, y con el carácter de extensión universitaria, en los años 1943 y 1944, la Universidad Central del Ecuador inauguró los "Primeros Cursos Libres de Periodismo", que se proponían suministrar conocimientos técnicos a los reporteros y más trabajadores intelectuales de la prensa nacional. Estos cursos contaron con ochenta alumnos. De esta manera se continuaban con los cursos en la Universidad Central del Ecuador (Pérez de Ricaurte, 1975, p. 1).

\section{Creación de la Escuela de Periodismo}

El 22 de junio de 1945, en la presidencia constitucional de Velasco Ibarra (1944-1947), la Asamblea Nacional, presidida por F. Arízaga Luque y cuyo Secretario General era Pedro Jorge Vera ${ }^{12}$, se decretó la creación de las Escuelas de Periodismo en las dos principales universidades de la República (Ecuador, 1945) ${ }^{13}$. "La Asamblea Constituyente Decreta: Art. 1 Crease en las Universidades de Quito y Guayaquil Escuelas de Periodismo en sus- titución del curso de Periodismo que ha venido funcionando en la Universidad Central. Art. 2 Para la organización y funcionamiento de dichas Escuelas, asignase la cantidad de cien mil sucres, que serán fijados en el presupuesto del Estado para el presente año. Estos fondos se repartirán en partes iguales entre las Universidades mencionadas en el Art 1. Art. 3 Por esta vez, a los alumnos matriculados en el primer curso de Periodismo, después de haber realizado el preparatorio, se les reconoce su situación de tales. Art. 4 Las citadas Universidades, elaboran de inmediato el Plan y los Programas de Estudios de dichas Escuelas. Art.5 Encargase de la Ejecución del presente Decreto, a los señores Ministros de Educación y Tesoro (Ecuador, 1945). Estos decretos se dieron en la ciudad de Quito, en la Sala de Sesiones de la Asamblea Nacional Constituyente, a los 8 días del mes de marzo de 1945 ". De esta manera quedan creadas las respectivas Escuelas de Periodismo. En el caso de la Escuela de Periodismo de la Universidad Central hasta inicios de la década de 1960 funcionó como parte de la Facultad de Pedagogía y Letras.

En 1963 se reconsideró su nombre y los abordajes académicos de la comunicación. Al inicio, la Escuela tenía un plan de estudios académicos humanísticos, de cuatro años, que aportaba conocimientos de cultura básica. Al finalizar este proceso se reconocía al estudiante con el título de Licenciado en Periodismo. En este periodo, el número de estudiantes no fue muy alto, pues no encontraban la satisfacción económica, ni tampoco la práctica de

12 En el año 1969, Pedro Jorge Vera, fue Subdirector de la Escuela de Ciencias de la Información.

13 En dicho registro se menciona que este decreto fue el 8 de marzo de 1945, para el caso de esta investigación más bien se retoma la fecha cuando fue expedido en el Registro Oficial, es decir el 22 de junio de 1945. 
la profesión. Además, los estudiantes sentían que los requisitos para obtener la licenciatura $^{14}$ eran muy exigentes. Esto provocó que durante el año 1955 se rediseñara el plan de estudios para que solo durase tres años la carrera ${ }^{15}$, a través de la simplificación y reducción de las materias. A partir de1963, la Escuela de Periodismo cambió su nombre a Escuela de Ciencias de la Información. En este cambio fue central el papel del Centro Internacional de Estudios Superiores de Comunicación para América Latina (CIESPAL), no solo porque su influencia fue decisiva en el abordaje, la concepción y el nombre de la Escuela, sino porque de alguna manera fue en la Universidad Central donde se creó esta institución.

\section{Escuela de Ciencias de la Información (1963- 1985)}

En 1959 se creó el CIESPAL: "El Consejo Universitario de la Universidad Central del Ecuador, el 29 de abril, presidido por el señor Doctor Alfredo Pérez Guerrero, en su calidad de Rector, resuelve la creación del Centro Internacional de Estudios Superiores de Periodismo para América Latina como un organismo universitario autónomo" (CIESPAL, 2019). De esta manera el papel de la comunicación da un giro muy importante pues la comunicación empieza a tener mucha relevancia, pero además la Universidad Central auspiciaba este nuevo momento.

Por otro lado, la dictadura militar (1963-1966) obstaculizaba el normal funcionamiento de las universidades en el país, en especial en la Universidad Central. "Durante ese tiempo las universidades fueron permanentemente hostigadas, en especial la Central. El gobierno realizó intervenciones; estudiantes, maestros y empleados fueron perseguidos y encarcelados. Además, aulas, bibliotecas y laboratorios fueron destruidos" (Universidad Central del Ecuador, 2012, p. 30). Es más, en esta década en varias ocasiones la universidad es clausurada. Tal como menciona Manuel Agustín Aguirre (1973) se dio un momento anti-universidad, al punto de denominar a la ley universitaria aplicada en eso años como "Carta Negra de la Esclavitud Universitaria”. El gobierno nombraba desde el rector hasta el último portero. Manuel Agustín Aguirre anota que: "desde el principio, las clausuras y reorganizaciones, permiten la infiltración por la ventana dictatorial, de un personal docente y aun administrativo, que forma un enclave castrense de la Universidad, para minarla y destruirla, una anti-Universidad, lo que engendra continuas contradicciones que agravaban la crisis" (Aguirre, 1973, p. 71).

14 Los requisitos para obtener la Licenciatura en Periodismo eran: acreditar 500 horas prácticas en un medio de comunicación social; presentar una tesis de grado sobre una de las asignaturas profesionales; preparar el diseño y diagramado de un periódico acompañado del material necesario para su publicación y el presupuesto correspondiente; someterse a un grado práctico sobre las técnicas de la profesión; presentarse al grado oral ante un tribunal integrado por cinco profesores. (Plan Director de Carrera, 1996:11).

15 Durante los 18 años, de la Escuela de Periodismo, existieron 3 planes de estudios diferentes. Sin embargo, los requisitos para la obtención del título eran las mismas. El primer plan duro diez años (con una tendencia humanística); el segundo duro cinco años, desde 1955 a 1960 (de carácter técnico- profesional, con tres años de estudio); y el tercero que fue desde 1960 a 1963 (mantuvo un equilibrio entre las materias de tendencia humanísticas y las de carácter técnico- profesional). (Plan Director de Carrera, 1996. Reforma Curricular. Ecuador: Facultad de Comunicación Social. Universidad Central del Ecuador). 
En medio de estas contradicciones, en que la universidad se batía en problemáticas internas y externas, en 1963, la Escuela de Ciencias de la Información se independizó de la Facultad de Filosofía, Letras y Ciencias de la Educación. Con toda la problemática que significó está separación y por las mismas circunstancias contextuales, las autoridades pensaron en la necesidad de un proceso para crear la Facultad de Comunicación Social. Dicha facultarización no fue posible, pero si un mayor nivel de autonomía al separarse de la Facultad de Filosofía.

La nueva escuela encontró en el CIESPAL su derrotero, pues esta institución, mediante múltiples charlas y seminarios, durante toda la década, dio a conocer las problemáticas propias de la comunicación en América Latina. Así, en 1965, esta institución realiza cuatro seminarios regionales, en los que se recomienda la transformación de las Carreras de Periodismo en "Institutos de Comunicación" o "Ciencias de la Información Colectiva". Los seminarios en CIESPAL permitieron un segundo momento de la comunicación, entendiéndola de forma académica y teórica, ya no solo desde la técnica periodística, sino también a partir de un fundamento teórico. En uno de estos seminarios, los participantes redactaron un "Plan Tipo" que fue recomendado a las diferentes escuelas y facultades de comunicación de la región. En Ecuador, la Escuela de Ciencias de la Información de la Universidad Central adoptó el "Plan Tipo" por pedido de los estudiantes y el Consejo Universitario, entonces dirigido por el rector, Francisco J. Salgado (FACSO, 1996). Siguiendo la nueva línea académica, la Escuela se separó de la Facultad de Filo- sofía, Letras y Ciencias de la Educación y se adscribió al rectorado; ello permitió que tuviera autonomía frente a las otras facultades de la universidad. Desde este momento, los estudiantes de la escuela ya no se formaron solo como periodistas, sino como comunicadores intelectuales, con perspectiva humanística (Fuentes Navarro, 1992).

El pensum académico, en ese momento, tenía sus bases en Lasswell, Lewin, Hovland, para el estudio de la comunicación a través de las "percepciones". Es decir, se definía al objeto de la comunicación con la fórmula "emisortransmisión-mensaje-canal-receptor”. Hasta entonces, el plan de estudio también tenía como base las teorías matemáticas: retroalimentación, entropía, ruido; las investigaciones tenían como temas: la publicidad y las relaciones públicas (Plan Director de Carrera, 2003. Reforma Curricular. Ecuador: Facultad de Comunicación Social. Universidad Central del Ecuador).

Sin embargo, a través del "Plan Tipo" se incorporó nuevas materias como: Teoría de la Comunicación, Investigación de la Comunicación, Filosofía, Sicología, Sociología, Relaciones Públicas, Diseño y Diagramación, Titulación, Historia Crítica del Ecuador, Investigación Científica de la Comunicación Colectiva, Técnica de los Medios Audiovisuales, Introducción a las Ciencias de la Información, entre otras. Con estas asignaturas se pretendía adaptar a la escuela los nuevos requerimientos de la comunicación moderna, con un componente humanístico y social.

Este nuevo abordaje de la comunicación pretendía un desarrollo técnico de los grandes medios de comunicación que, en ese momento, demandaban un nuevo 
profesional técnico y con criterio teórico. El aumento de materias teóricas se vio influido por el proceso histórico-político que la región afrontaba en aquella época marcada por las dictaduras militares y la implementación de medidas de recorte de gasto público y privatización de diferentes sectores estratégicos (la comunicación se vio afectada por la falta de insumos tecnológicos y laboratorios prácticos de investigación); lo que hacía necesario conocer más de cerca la teoría histórica y política.

La ausencia de un presupuesto adecuado para la educación, influyó también en la construcción o adecuación de infraestructuras de las escuelas o facultades. En el Ecuador, la Escuela de Ciencias de la Información no tuvo apoyo económico para construir su propio edificio en esos años. En principio, se trasladó a una casa particular en la avenida América; posteriormente se ubicó en otra casa en la calle Versalles. $\mathrm{Su}$ último cambio fue al último piso del edificio de Servicios Generales, dentro del campus universitario. Las dificultades que pasó la Escuela para obtener un edificio propio muestran el insuficiente apoyo de las autoridades para la formación de comunicadores en el país (FACSO, 1996). Recién en la década del setenta se asignó presupuesto para la construcción de un edificio propio y de centros técnicos de trabajo y su equipamiento. "En 1975, la escuela contaba con un local reducido, una pequeña biblioteca, una sala de redacción con 40 máquinas, un pequeño laboratorio de fotografía, un mimeógrafo, 10 máquinas fotográficas, equipo de videotape, grabadoras, mesas de diagramación y otros equipos menores" (Pérez de Ricaurte, 1975, p. 9).
En los dieciséis años de existencia de la Escuela, se utilizaron diez diferentes planes de estudio. El "Plan Tipo" fue el que tuvo mayor permanencia y estuvo vigente casi diez años. Del resto de planes de estudios, dos son más significativos: el primero, de 1971, establece por primera vez la especialización en cuarto año. Los estudiantes podían optar entre cuatro especialidades: Impreso, Audiovisual, Relaciones Públicas y Publicidad. El segundo se implementó en 1975 y acogió las sugerencias hechas por el Seminario de Escuelas de Ciencias de la Comunicación, realizado en Costa Rica, en 1973, por CIESPAL. En esta época, CIESPAL realizó una revisión de investigaciones a nivel regional que permitió recopilar datos en la parte teórica-metodológicas del período 60-70. La corriente funcionalista norteamericana de la Mass Communication Research fue la que tuvo mayor impacto, seguida -en menor medida- de la corriente semiótica europea de análisis de contenido. Entre los temas de investigación se encontraron: la historia, la legislación, el análisis de contenido en prensa, la comunicación para el desarrollo rural y lo referido a la estructura, funciones y efectos de los medios en la audiencia (León, 2012).

El paradigma de las nuevas investigaciones fue el norteamericano, pero influido por el pensamiento económico de América Latina, en auge en ese momento. La economía se entendía no desde la mirada del exterior sino desde las demandas regionales que apoyó la Comisión Económica para América Latina y el Caribe (CEPAL). Esta comisión también favoreció el nuevo desarrollo y abordaje de la comunicación creado desde la región a partir de la comunicación para el desarrollo, la teoría crítica y el estructuralismo. Esta nueva visión de la comunicación 
trata de romper con la economía y el pensamiento hegemónico norteamericano para proponer nuevas bases teórico-metodológicas que se ajusten a la realidad latinoamericana.

De hecho, se ha podido constatar que desde la década de 1960 hay referencias explícitas al papel de los medios de comunicación en la promoción del desarrollo. Además, se empiezan a perfilar críticas a las teorías funcionalistas de la comunicación, en concordancia directa con la ruptura ideológica contra el proyecto hegemónico norteamericano. Como apoyo a esta ruptura, los teóricos de la CEPAL introducirían el concepto de planificación, que se tornaría un componente clave en los estudios de comunicación en la región desde inicios de los años setenta del siglo pasado (León, 2012, p. 245).

Además, en esta década, aparece la figura del comunicólogo como científico social. Aunque no en todos los casos, sí en la mayoría de los diseños curriculares que adoptaron este modelo se dio más relevancia a la teoría crítica, es decir, de materialismo histórico, economía política y otros contenidos marxistas y se desplazó la formación y la habilitación profesional. Más allá de algunos casos notables de desarrollo de este modelo, hay un conjunto de rasgos muy generalizados asociados a él. Uno es el teoricismo y su reacción inmediata, el practicismo, es decir, la oposición maniquea entre la teoría -que llegó a ser reducida a unos cuantos dogmas religiosamente consagrados- y la práctica que, a su vez, llegó a reducirse a la reproducción de algunos estereotipos de los medios masivos (Fuentes Navarro, 1992, p. 5).

En este sentido, los años setenta marcaron un desarrollo en la comunica- ción, sobre todo desde Latinoamérica, lo que provocó frecuentes cambios en las mallas curriculares. La comunicación a nivel macro cambiaba, por lo tanto, debía cambiar a nivel institucional educativo. Desde 1972 hasta 1975, la Escuela de Ciencias de Información de la UCE tuvo varias modificaciones. El plan de especializaciones vigente de 1972 a 1973 no tuvo éxito, por lo que fue reemplazado por uno que estaba conformado por un 56\% de materias técnico-profesionales y del campo humanístico 44\%. Desde 1973 a 1974 el plan de estudios sufrió pequeñas modificaciones en la cantidad de materias humanísticas; el plan de 1974-1975 muestra un incremento de cátedras, existiendo un $43 \%$ de asignaturas humanísticas y 53\% de materias profesionales. En el plan que se ejecutó entre los años 1975 a 1978 existe un $65 \%$ de materias técnico-profesionales y sólo un 35\% del área humanística (Facultad de Comunicación Social, 2017:12).

Según un estudio realizado por la Federación Latinoamericana de Facultades de Comunicación Social (FELAFACS), en las facultades y escuelas de comunicación, a nivel regional, un 40\% de las materias trataban sobre teorías e investigación, por ejemplo, Teorías de la Comunicación, Lenguajes y Estéticas y Metodologías de Investigación. El 20\% de las materias tenía temas sobre Legislación, Ética y Política, Comunicación y Desarrollo, y Comunicación y Cultura. Finalmente, el 40\% restante de las materias estaban relacionadas con el ejercicio profesional práctico (Fuentes Navarro, 1992).

A partir de esto, los estudiantes de la Escuela de Ciencias de la Información de la UCE exigieron que los enfoques académicos se formularan de acuerdo al pro- 
ceso de industrialización, político y sociocultural, que vivía el Ecuador de ese entonces. Por lo tanto, la reforma teórica del pensum académico fue necesaria, sobre todo por una nueva corriente que se generaba en América Latina contra el funcionalismo. En esta época surgieron materias teóricas encabezadas por los siguientes autores: Armand Mattelart, Antonio Pasquali, Eliseo Verón, que aportaban a la Teoría de la Comunicación crítica a la corriente funcionalista. Así, el abordaje académico tomaba en consideración la denominación cultural, y la realidad circunstancial de los países subdesarrollados (FACSO, 2003).

En este sentido, los estudiantes demandaron la formación de un "verdadero comunicador" y que existiera un proceso de facultarización de la Escuela. Producto de estas demandas, en 1978, la Escuela dio el primer paso para ser facultad. Hubo una apertura al diálogo sobre propuestas y opiniones de los estudiantes para el rumbo de la facultad y tuvo lugar una implementación de nuevos equipos, entre ellos un set de televisión, el taller de imprenta y laboratorios de fotografía. Además, en 1979 se implementan las prácticas pre-profesionales como parte formativa de la carrera (La comunicación Social en el Ecuador: Historia de la Facultad de Comunicación Social de la Universidad Central del Ecuador. Informe. Ecuador: Facultad de Comunicación Social). Hasta este período, la Escuela tuvo 13 planes académicos. El último plan ya mostraba un mayor énfasis en la tendencia de formar comunicadores sociales y no solo periodistas.

En 1980, por primera vez, los estudiantes logran escoger la especialidad de su agrado, lo que no sucedía con las mallas anteriores. La mayoría de estudiantes se graduó con procesos de titulación de grado oral y elaboración de un trabajo práctico. Egresaron cuatrocientos diecinueve estudiantes $(37,4 \%$ del total de alumnos) y se titularon trescientos veintisiete (29,1\% del total). Los egresados, de ese entonces no consideraban como mejor opción la producción de trabajos científicos e investigativos (FACSO, 1996).

En conclusión, los cambios más importantes en la Escuela se dan a partir de la sugerencia de CIESPAL de abordar la comunicación desde América Latina y no solo vinculada con la teoría norteamericana y con los objetos de estudios de los mass media o medios de comunicación. Este criterio pretendía mejorar y aumentar la investigación con nuevas bases teóricas, sobre todo de la corriente crítica y el desarrollo social. Hacia inicios de los ochenta, en la Escuela de Ciencias de la Información se empieza a introducir el Estructuralismo como una corriente analítico-formativa en el estudio de la lengua materna, y en la comprensión de otros sistemas de comunicación; además, en la enseñanza de la gramática estructural en la educación media, y la circulación de ciertas obras de autores célebres como Roland Barthes. El estudio y la comprensión del lenguaje verbal y de otros sistemas no verbales se vieron muy favorecidos por la introducción de la asignatura de Semiótica y Semántica, que si bien, al principio tuvo una clara filiación saussureana, lentamente se adecuó a contenidos de las diversas corrientes del dominio semio-lingüístico. Referentes de lo afirmado son autores como Hjelmslev, Jakobson, Martinet, Ullmann, Chomsky, Pierce, Morris, Barthes, Todorov, Eco, Greimas (FACSO, 2003, p. 19). 


\section{Facultad de Comunicación Social (FACSO) de la Universidad Central del Ecuador (1983-actualmente)}

El 24 de octubre de 1983, el Congreso Nacional, por el Decreto No.145, asignó un presupuesto anual para las facultades de comunicación social, tanto en la Universidad Central del Ecuador como en la Universidad Estatal de Guayaquil. El porcentaje del valor asignado fue del 3\% del rendimiento total del impuesto del 1\% a las ventas de divisas extranjeras en el mercado libre (FACSO, 1996). Ello permitió mejorar e implementar recursos para los nuevos profesionales. Además, facilitó la restructuración de la Escuela de Ciencias de la Información para convertirla en facultad.

Para 1984, los estamentos de la Escuela de Ciencias de la Información, resolvieron iniciar la reforma académica y administrativa, luego de un largo proceso de debate alrededor de la idoneidad de la formación que se brindaba a los estudiantes. Esta corriente determinó la necesidad de modificar la estructura de la Escuela de Ciencias de la Información, para constituirla en Facultad. Para llevar adelante este proceso se dieron sucesivas reuniones de los organismos de gobierno de la Escuela que concluyeron en la decisión de nombrar la Comisión de "Facultarización”, como el organismo encargado de preparar los informes correspondientes de sustento y gestionar la creación de la Facultad de Comunicación Social (FACSO, 1996, p. 15).
El trabajo de la Comisión empezó en noviembre de 1983 y finalizó el 5 de febrero de 1985 con la aprobación del Consejo Universitario para la creación de la facultad. Las autoridades cambiaron el nombre con la finalidad de mostrar el "verdadero significado de la comunicación"16. Este cambio, correspondió al último modelo de comunicación, que busca la formación de comunicadores ubicados en América Latina, que puedan comprender sus realidades y, a partir de ellas, investigar y solventar los problemas de comunicación. El objetivo general de la creación de la Facultad es "formar el recurso humano que logre plasmar en su ejercicio profesional tales concepciones y enfoques básicos, como los mencionados, acerca de la comunicación" (FACSO, 1996, p. 16).

En este sentido, la reforma del pensum académico tuvo un nuevo discurso hacia la Comunicación Social. Se modificó el plan académico en dos etapas. La primera era el Ciclo Común y correspondía a los tres primeros años con materias que permitieron mayor reflexión teórica de la Comunicación Social como: Comunicación y Desarrollo, Comunicación y Problemas Sociales, Comunicación Alternativa, Planificación de la Comunicación. La segunda etapa era el último año donde los estudiantes escogían su especialidad. Con este concepto de educación especializada se abrió cuatro especialidades: Comunicación Impresa, Televisión, Radio, Comunicación General e Institucional e Investigación de la Comunicación.

16 En este momento es cuando se concibe a la comunicación como una disciplina social de investigación y se critica el reduccionismo de asociar comunicación y periodismo. "Se entendía, entonces, que la comunicación es un proceso racional de interacción entre los sujetos sociales, es una herramienta que motiva y orienta la participación, la reflexión crítica y el dialogo entre los diferentes sujetos sociales en todos los ámbitos de actividad humana y que considera las formas y niveles de asociación humana, bajo el propósito de lograr el cambio de las estructuras" (Plan Director de Carrera, 1996:15). 
En 1996 se realizó la segunda reforma del pensum académico que inclinó la formación de la FACSO hacia la Escuela de Palo Alto y sus matrices epistemológicas $^{17}$. Los aportes teóricos más influyentes fueron de autores clásicos y contemporáneos como: R.L. Birdwhistell, E. Goffman, E. Hall, F. Davis, L.M. Knapp, entre otros. La malla cambió los cuatro años de estudio por ocho semestres académicos, es decir, en este momento se pasó de la modalidad basada en años a la de semestres. Las materias Teoría y Práctica de la Comunicación, Comunicación como Proceso, e Ideología de la Comunicación abrieron nuevos enfoques de la comunicación, sobre todo críticos, con autores como: Martín Barbero, Verón, Mattelart, Althusser, Foucault, Propp, Lotman, Baudrillard y Habermas. En aquella época se admitió estudios teórico-prácticos y con un proceso de titulación donde se elabora, de forma obligatoria, Tesis de Grado de Investigación o de Producción, calificada y aprobada mediante la defensa ante un tribunal. Con este proceso realizado se obtenía el título de Licenciado en Comunicación Social, con mención en una de las especialidades (FACSO, 1996).

Posteriormente, se realizó una nueva restructuración del pensum académico en el año 200o. Esta malla comprende que la Comunicación Social se articula con el proceso de la producción social y se entiende como elemento de construcción económico, político, social y cultural. En esta etapa, la base teórica está marcada por la corriente marxista y la Escuela de Frankfurt. Además, se eliminan los énfasis y los profesionales se graduaban con el título de Licenciado en Comunicación Social.

Estas teorías estuvieron muy marcadas hasta el año 2017. Cabe anotar que se produjo otra reforma en 2008 donde el primer período era de primero a séptimo semestres con materias de tronco común; y la segunda etapa de octavo a noveno semestre con énfasis de profesionalización en tres áreas: Comunicación Organizacional, Periodismo, y Educomunicación, Arte y Cultura. El peso de la malla curricular caía en las Teorías de la Comunicación y Materias de Investigación, pero las materias dedicadas al área de historia también tuvieron importancia. A partir del 2012, con la reforma de la Ley Orgánica de Educación Superior (LOES), se institucionalizó el portafolio y syllabus de todas las materias en la carrera ${ }^{18}$.

En la actualidad, el pensum académico de la FACSO funciona con su última reforma en el año 2017. Este tiene como base ajustarse a las demandas laborales actuales, de la mano de lo académico ${ }^{19}$. En este sentido, eliminó los tres énfasis anteriores y elaboró dos énfasis nuevos.

17 Los enfoques epistémicos de la Escuela de Palo Alto refieren "a la denominada comunicación orquestal, que implica la inserción de los individuos en sus relaciones familiares, grupales, institucionales, étnicas; con base en el contexto sociocultural, en donde la objetualidad, vestimenta, cinésica, prosémica, temporalidad, cromática y cotidianidad, en general, son sus objetos de estudio preferentes" (Plan Director de Carrera, 2003:19).

18 El portafolio es un registro de los docentes para verificar el sistema de evaluación, donde se implementó el examen de reactivos. El syllabus, por su parte, es el documento que el docente le entrega al estudiante donde se coloca el objetivo de la materia, base teórica de la materia, textos, autores, entre otros. Esto permitió mejorar la organización del sistema académico universitario en el país y la acreditación de las universidades como tal.

19 Entrevista. Luis Molina, ex Director de Carrera de la Facultad de Comunicación Social. Universidad Central del Ecuador. 
Ahora, los estudiantes, a partir de quinto semestre toman su especialidad en Gestión de la Comunicación o Periodismo y Narrativas Mediáticas. En esta malla aumentaron materias de profesionalización, sobre todo, en el periodismo digital para el énfasis de Periodismo y Narrativas Mediáticas y sobre estrategias y herramientas digitales de la comunicación en el énfasis de Gestión de la Comunicación. Esta malla retoma la teoría de la información, pero no deja de lado la teoría de la cultura e industrias culturales, posiciona una base teórica y profesional complementaria al sistema actual que vivimos a través de la globalización.

Finalmente, la construcción de la FACSO tuvo varias etapas, desde la Escuela de Periodismo hasta la conformación de la Facultad. Asimismo, han pasado 18 planes académicos aplicados a lo largo de la historia de la FACSO y, con ello, discursos distintos del concepto y la práctica de la comunicación y la información.

\section{Consideraciones finales}

Como se puede observar, a lo largo del artículo se pretende mostrar la genealogía de la Facultad de Comunicación Social de la Universidad Central del Ecuador. Lo primero que se cuestiona es el momento de su creación, casi siempre situado por los estudiosos en la década del cuarenta (1945). En base a los archivos revisados se puede afirmar que las discusiones sobre la creación de la $\mathrm{Ca}$ rrera de Periodismo se dieron desde finales de la década del treinta del siglo pasado. En ese sentido, este escrito coloca nueva información corroborada en la revisión de los archivos del HCU. Cabe recalcar que el trabajo de archivo recién inicia, bien se puede afirmar que ahí se encuentran verdaderas "joyas" históricas. Por ello es indispensable considerar la digitalización de todos los archivos universitarios, similar a la que muchas universidades en la región lo han hecho, pues solo a través de esa medida se podría profundizar el trabajo investigativo.

También es importante volver sobre la comunicación como una disciplina en constante cambio y esto sólo se puede analizar en las mallas curriculares que se han ejecutado a lo largo de la historia de la FACSO. En este sentido, la investigación de la estudiante Ariana Román es indispensable; sin embargo, es necesario que más investigadores indaguen en las mallas curriculares; en la búsqueda de indicios que permitan conocer los giros académicos y epistémicos que esta ha tenido.

Finalmente, es preciso reconocer que un trabajo con tal nivel de esfuerzo solo es posible con a la participación de las y los estudiantes, núcleo de la universidad, pues gracias a su aporte es posible seguir investigando. 


\section{Bibliografía}

Aguirre, M. A. (1973). La segunda Reforma Universitaria. Editorial Universitaria.

CIESPAL. (2019). Historia. CIESPAL. https://ciespal.org/historia/

Ecuador. (1945, junio 22). Decreto de la creación de las Escuelas de Periodismo en las dos principales universidades de la República. Registro Oficial.

Escobar, V. H., \& Ramírez, E. (1993). FACSO Pasado y Presente. Universidad Central del Ecuador.

FACSO. (1996). Plan Director de la Carrera de Comunicación Social. Universidad Central del Ecuador.

FACSO. (2003). Plan Director de la Carrera de Comunicación Social. Universidad Central del Ecuador.

Fuentes Navarro, R. (1992). El estudio de la comunicación desde una perspectiva sociocultural en América Latina. Diálogos de la comunicación, 32. https://dialnet.unirioja.es/servlet/articulo?codigo=2700942

García, J. (2005). 60 años de trayectoria: Historia de la FACSO. Textos y Contextos, 4, 125-136.

Hallo, W. (1992). Síntesis histórica de la comunicación y el periodismo en el Ecuador. Ediciones El Sol. http://biblioteca.udla.edu.ec/client/en_US/default/search/detailnonmodal/ent:\$002f\$002fSD_ILS\$002f12\$002f

SD_ILS:12341/ada?qu=HISTORIA+--+ECUADOR\&ic $=$ true\&te $=$ ILS\&ps $=300$

León, G. (2012). El papel de Ciespal en el proceso de institucionalización de los estudios de la Comunicación en América Latina: History and Communication in Latin America: The Ciespal role in the institutionalization of communication studies in Latin America. Miguel Hernández Communication Journal, 3, 235-261.

Maldonado, F. (2009). La trayectoria de la Facultad y de la Comunicación en la Historia Nacional. Textos y Contextos, 8, 69-74.

Pérez de Ricaurte, A. (1975). Planes de estudio. Universidad Central del Ecuador.

Román, A (2020). Tesis de grado Análisis de la transformación del discurso comunicacional en los tres últimos pensum académicos de la Facultad de Comunicación Social de la Universidad Central del Ecuador

Acta de Sesión Honorable Consejo Universitario de 14 de noviembre de 1938 A, (1938) (Universidad Central del Ecuador).

Acta de Sesión Honorable Consejo Universitario del 14 de noviembre de 1938 B, (1938) (Universidad Central del Ecuador).

Acta de Sesión Honorable Consejo Universitario del 25 de noviembre de 1938 A, (1938) (Universidad Central del Ecuador).

Acta de Sesión Honorable Consejo Universitario del 25 de julio de 1944 A, (1944) (Universidad Central del Ecuador).

Acta de Sesión Honorable Consejo Universitario del 25 de septiembre de 1944 A, (1944) (Universidad Central del Ecuador).

Universidad Central del Ecuador. (2012). Protagonistas de la historia. Editorial Universitaria. 